\title{
Compact explicit matrix representations of the flexoelectric tensor and a graphic method for identifying all of its rotation and reflection symmetries
}

\author{
H. Le Quang ${ }^{1, a)}$ and Q.-C. He $\mathrm{e}^{1,2}$ \\ 1) Université Gustave Eiffel, CNRS, MSME UMR 8208, F-77454 Marne-la-Vallée, \\ France. \\ 2) Southwest Jiaotong University, School of Mechanical Engineering, Chengdu 610031, \\ PR China.
}

(Dated: 17 May 2021)

Flexoelectricity is an electromechanical phenomenon produced in a dielectric material, with or without centrosymmetric microstructure, undergoing a non-uniform strain. It is characterized by the fourth-order flexoelectric tensor which links the electric polarization vector with the gradient of the second-order strain tensor. Our previous work [Le Quang and He, Roy. Soc. London, Ser. A 467, 2369-2386, 2011] solved the fundamental theoretical problem of determining the number and types of all rotational symmetries that the flexoelectric tensor can exhibit. In the present one, compact explicit matrix representations of the flexoelectric tensor are provided so as to facilitate the use of it with any possible rotational symmetry. The number and types of all reflection symmetries that the flexoelectric tensor can have are also determined. To identify the rotational symmetry and reflection symmetry of a given flexoelectric tensor, a simple and efficient graphic method based on the concept of pole figures is presented and illustrated.

PACS numbers: Valid PACS appear here

\section{INTRODUCTION}

Flexoelectricity is a coupled electromechanical phenomenon appearing a dielectric material subjected to a non-uniform strain. In contrast to piezoelectricity, it can be generated even in a dielectric material whose microstructure is centrosymmetric. Indeed, in the case of a dielectric material undergoing a uniform strain, the electric polarization is produced if and only if the microstructure of this material is non-centrosymmetric. However, when a dielectric material with (or without) a centrosymmetric microstructure is subjected to a nonuniform strain whose gradient is non null, a relative displacement of the centers of the positive and negative charges is resulted in and gives rise to an electric polarization.

The flexoelectric effects can be produced in a multitude of situations, for example, in bending crystal plates ${ }^{1}$, nanobeams and nanowires 24 or when stretching thin films ${ }^{5}$ on liquid crystals ${ }^{6}$ and on elastomers ${ }^{7}$. The flexoelectric constants describing the flexoelectric effects of some dielectric materials were observed and measured in a direct or indirect way in a few experimental works such as those made by $\mathrm{Ma}$ and Cross ${ }^{8-10}$ and Zubko et $a l l^{[11}$ for various perovskites which exhibit unusually high flexoelectricity, the ones of Kalinin and Meunier ${ }^{12}$ and Naumov et al! $\frac{13}{13}$ for low-dimensional structures like nanographitic systems and two-dimensional boronnitride sheets or by Zhang et al ${ }^{14115}$, Chu and Salem ${ }^{16}$ and Zhou et al ${ }^{17}$ for dielectric materials and polymers

\footnotetext{
a) Corresponding author

Tel: 33 (0) 160957 797; Fax: 33 (0) 160957 799;

Email: hung.le-quang@univ-eiffel.fr
}

such as $\mathrm{TiO}_{2}$ ceramics and the polyvinylidene fluoride (PVDF). In parallel with these experimental works, theoretical studies were also conducted to demonstrate the size-dependent flexoelectric properties and surface effect of dielectric materials/structures in nanoscale, for example, by Sahin and Dost $\frac{18}{18}$, Tagantse $\sqrt{19120}$, Yurkov and Tagantsev ${ }^{21}$, He et al ${ }^{[22}$, Qi et al ${ }^{[23}$ and Bai et al.$^{24}$. Numerical approaches were elaborated according either to the first-principles method, for example, by Maranganti and Sharma ${ }^{25}$, Hong et al. ${ }^{26 \mid 27}$ or to the other theoretical calculation methods such as finite element method by Deng et al ${ }^{28}$ and Yvonnet et al ${ }^{29}$, phase-field method by Li et al ${ }^{30}$ and Wang et al ${ }^{31}$ to estimate the flexoelectric properties of some dielectric materials/structures. For more references about flexoelectricity and for discussions on potential important applications of flexoelectricity, the reader is referred to Tagantsev et al ${ }^{[20}$, Sharma et $a l l^{32}$, Zubko et al. ${ }^{[33}$, Wang et al! $!^{34}$, Narvaez et al ${ }^{[35}$, Abdollahi et al. ${ }^{36}$ and Shu et al. ${ }^{[37 / 38}$.

When a dielectric material is subjected to small deformations and when the piezoelectric and flexoelectric phenomena produced in it are linear, the electric polarization vector $\mathbf{p}$ is related to the infinitesimal strain tensor $\varepsilon$ and the gradient of the latter, namely $\mathbb{E}=\nabla \varepsilon$, by a linear relation:

$$
p_{i}=D_{i j k} \varepsilon_{j k}+F_{i j k l} E_{j k l} .
$$

Above, $D_{i j k}$ are the matrix components of the thirdorder piezoelectric tensor $\mathbb{D}$ and $F_{i j k l}$ stand for the matrix components of the fourth-order flexoelectric tensor $\mathbb{F}$. Due to the symmetry $\varepsilon_{i j}=\varepsilon_{j i}$ of $\varepsilon$, the strain gradient $\mathbb{E}$ possesses the property $E_{i j k}=E_{j i k}$ and the matrix components of $\mathbb{F}$ have the following index permutation symmetry:

$$
F_{i j k l}=F_{i k j l} .
$$


Note that, if the microstructure of the dielectric material in question exhibits centrosymmetry, the requirement that the third-order tensor $\mathbb{D}$ be invariant under the central inversion transformation implies that $\mathbb{D}$ is null, so that the constitutive law (1) reduces to

$$
p_{i}=F_{i j k l} E_{j k l} \text {. }
$$

In other words, when the dielectric material has a centrosymmetric microstructure, the piezoelectric effect disappears and the electric polarization vector $\mathbf{p}$ depends only on the strain gradient $\mathbb{E}$.

In the linear constitutive law (1), the classical thirdorder piezoelectric tensor $\mathbb{D}$ has been completely investigated and understood; however, the fourth-order flexoelectric tensor $\mathbb{F}$, which is much more complicated than the usual fourth-order elastic tensor, is far from being thoroughly studied and understood. In our previous one $e^{39}$, the number and types of all possible rotational symmetries for the flexoelectric tensor $\mathbb{F}$ were specified and the number of independent material parameters of $\mathbb{F}$ belonging to each possible symmetry class was determined. Later, Shu et al.$^{40}$ gave the matrix representations of $\mathbb{F}$ for various symmetries.

The present work can be regarded as a continuation of our previous one $e^{39}$. Precisely, novel $3 \times 18$ matrix representations of the flexoelectric tensor $\mathbb{F}$ are provided for all 12 rotational symmetries determined in our previous study 239 . The matrix representations of $\mathbb{F}$ given in the present work are well-structured and much more com-

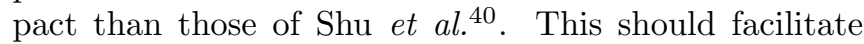
the practical use of $\mathbb{F}$ in various anisotropic cases. In addition, the flexoelectric tensor $\mathbb{F}$ is further investigated in the present work by determining all of its reflection symmetries. It is proved that the 12 rotational symmetry classes of the flexoelectric tensor $\mathbb{F}$ are reduced to 8 reflection symmetries and these 8 reflection symmetries are identical to those of the fourth-order elastic tensor. Finally, a simple but efficient graphic method based on the notion of pole figures is suggested and illustrated for identifying the reflection symmetry and rotational symmetry that a given flexoelectric tensor may have.

The paper is structured as follows. In Section II, some notations and definitions used throughout the paper are presented. In Section III, the main results obtained in our previous work ${ }^{39}$ on the symmetry groups and symmetry classes of the flexoelectric tensor are recalled for the paper to be self-contained. In Section IV, compact explicit matrix representations of the flexoelectric tensor for all possible rotational symmetries are provided. Section $\mathrm{V}$ is dedicated to finding out the reflection symmetry classes of the flexoelectric tensor. In Section VI, a simple and efficient graphic method is elaborated to identify not only the reflection symmetry but also the rotational symmetry of a given flexoelectric tensor. In Section VII, some concluding remarks are given.

\section{NOTATIONS AND DEFINITIONS}

Let $\mathcal{V}$ be a three-dimensional (3D) inner-product space over the reals $\mathcal{R}$ and Lin be the space of all linear transformations (second-order tensors) on $\mathcal{V}$. The inner product of two vectors $\mathbf{a}$ and $\mathbf{b}$ of $\mathcal{V}$ is symbolized by $\mathbf{a} \cdot \mathbf{b}$. The $3 \mathrm{D}$ orthogonal group $O(3)$ is defined as $O(3)=\{\mathbf{Q} \in \operatorname{Lin} \mid \mathbf{Q} \mathbf{a} \cdot \mathbf{Q b}=\mathbf{a} \cdot \mathbf{b}, \forall \mathbf{a}, \mathbf{b} \in \mathcal{V}\}$. The $3 \mathrm{D}$ rotation group $S O(3)$ is given by $S O(3)=\{\mathbf{Q} \in$ $O(3) \mid \operatorname{det} \mathbf{Q}=1\}$. In what follows, $\mathbf{Q}(\mathbf{a}, \theta)$ stands for the rotation about $\mathbf{a} \in \mathcal{V}$ through an angle $\theta \in[0,2 \pi)$. In particular, $\tilde{\mathbf{Q}}, \hat{\mathbf{Q}}$ and $\mathbf{\mathbf { Q }}$ denote, respectively, the rotations $\mathbf{Q}\left(\mathbf{e}_{1}+\mathbf{e}_{2}+\mathbf{e}_{3}, 2 \pi / 3\right), \mathbf{Q}\left(2 \mathbf{e}_{2}+\varphi^{2} \mathbf{e}_{3}, 2 \pi / 3\right)$ and $\mathbf{Q}\left(\varphi \mathbf{e}_{2}+\mathbf{e}_{3}, \pi\right)$ with the golden ratio $\varphi=(1+\sqrt{5}) / 2$.

For later use, it is convenient to introduce the following standard group notations:

(i) the identity group, denoted by $I$, is formed by the second-order identity tensor $\mathbf{I}$;

(ii) the cyclic group $Z_{r}(r \geq 2)$ contains $r$ elements generated by $\mathbf{Q}\left(\mathbf{e}_{3}, 2 \pi / r\right)$. In particular, when $r \rightarrow \infty$, $Z_{r}$ becomes the group $S O(2)$ consisting of all rotations $\mathbf{Q}$ about $\mathbf{e}_{3}$ such that $\mathbf{Q} \mathbf{e}_{3}=\mathbf{e}_{3}$;

(iii) the dihedral group $D_{r}(r \geq 2)$ comprises $2 r$ elements generated by $\mathbf{Q}\left(\mathbf{e}_{3}, 2 \pi / r\right)$ and $\mathbf{Q}\left(\mathbf{e}_{1}, \pi\right)$. The corresponding form of $D_{r}$ when $r \rightarrow \infty$ is $O(2)$ consisting of all orthogonal tensors $\mathbf{Q}$ such that $\mathbf{Q} \mathbf{e}_{3}= \pm \mathbf{e}_{3}$;

(iv) the spatial groups $\mathcal{T}, \mathcal{O}$ and $\mathcal{I}$ with $\mathcal{T}$ representing the tetrahedral group of 12 elements generated by $D_{2}$ and $\tilde{\mathbf{Q}}, \mathcal{O}$ being the octahedral group of 24 elements generated by $D_{4}$ and $\tilde{\mathbf{Q}}$, and $\mathcal{I}$ symbolizing the dodecahedral group of 60 elements generated by $D_{5}, \hat{\mathbf{Q}}$ and $\mathbf{Q}$. Recall that the spatial groups $\mathcal{T}, \mathcal{O}$ and $\mathcal{I}$ map a tetrahedron, a cube and a dodecahedron onto themselves, respectively.

Next, we define the space of flexoelectricity tensors as follows:

$$
\mathcal{F}=\left\{\mathbb{F}=F_{i j k l} \mathbf{e}_{i} \otimes \mathbf{e}_{j} \otimes \mathbf{e}_{k} \otimes \mathbf{e}_{l} \mid F_{i j k l}=F_{i k j l}\right\}
$$

The symmetry group of a flexoelectricity tensor $\mathbb{F} \in \mathcal{F}$, is denoted by $\mathcal{G}(\mathbb{F})$ and characterized as

$$
\mathcal{G}(\mathbb{F})=\{\mathbf{Q} \in S O(3) \mid \mathbf{Q} * \mathbb{F}=\mathbb{F}\}
$$

where $\mathbf{Q} * \mathbb{F}=Q_{i r} Q_{j m} Q_{k n} Q_{l s} F_{r m n s} \mathbf{e}_{i} \otimes \mathbf{e}_{j} \otimes \mathbf{e}_{k} \otimes \mathbf{e}_{l}$. The definition of $\mathcal{G}(\mathbb{F})$ implies that $\mathcal{G}(\mathbb{F})$ is a closed subgroup of $S O(3)$ and $\mathcal{G}(\mathbf{Q} * \mathbb{F})=\mathbf{Q} \mathcal{G}(\mathbb{F}) \mathbf{Q}^{T}$ for any orthogonal tensor $\mathbf{Q} \in O(3)$. On the other hand, $\mathbb{F}$ is said to exhibit $G$-symmetry when $G \subseteq \mathcal{G}(\mathbb{F})$ and $\mathbf{Q} * \mathbb{F}=\mathbb{F}$ for all $\mathbf{Q} \in$ $G$. Consequently, two materials characterized by their respective flexoelectric tensors $\mathbb{F}_{1} \in \mathcal{F}$ and $\mathbb{F}_{2} \in \mathcal{F}$ are said to have the same type of symmetry if and only if the symmetry groups of $\mathbb{F}_{1}$ and $\mathbb{F}_{2}$ are conjugate to each other, i.e.

$$
\mathbb{F}_{1} \sim \mathbb{F}_{2} \Leftrightarrow \mathcal{G}\left(\mathbb{F}_{1}\right) \sim \mathcal{G}\left(\mathbb{F}_{2}\right) \Leftrightarrow \exists \mathbf{Q} \in S O(3) \text { such that } \mathcal{G}\left(\mathbb{F}_{1}\right)=\mathbf{Q} \mathcal{G}\left(\mathbb{F}_{2}\right) \mathbf{Q}^{T}
$$


TABLE I. List of notations

\begin{tabular}{|c|c|}
\hline Notations & Descriptions \\
\hline $\mathbf{p}, p_{i}$ & Electric polarization vector and its components \\
\hline$\underset{\sim}{\mathbb{F}}, F_{i j k l}$ & Fourth-order (type-II) flexoelectric tensor and its components \\
\hline$\tilde{\mathbf{F}}, \tilde{F}_{i \alpha}$ & Matrix representation of the flexoelectric tensor and its components \\
\hline $\mathbb{F}^{I}, F_{i j k l}^{I}$ & Fourth-order type-I flexoelectric tensor and its components \\
\hline $\mathbb{D}, D_{i j k}$ & Third-order piezoelectric tensor and its components \\
\hline$\varepsilon, \varepsilon_{i j}$ & Infinitesimal strain tensor and its components \\
\hline$\underset{\sim}{\mathbb{E}}, E_{\sim j k}$ & Strain gradient tensor and its components \\
\hline$\tilde{\mathbf{E}}, \tilde{E}_{\alpha}$ & Vector representation of the strain-gradient tensor and its components \\
\hline $\mathbf{H}, \mathbb{H}, \mathbb{H}$ & Second-, third-, fourth-order harmonic tensors \\
\hline $\mathcal{F}$ & Space of flexoelectricity tensors \\
\hline Q & Generic orthogonal tensor \\
\hline $\mathbf{Q}(\mathbf{a}, \theta)$ & Rotation about a through an angle $\theta$ \\
\hline $\mathcal{G}(\mathbb{F})$ & Rotation symmetry group of $\mathbb{F}$ \\
\hline$\left\{\mathcal{G}_{i}\right\}$ & Rotation symmetry class \\
\hline$Z_{n}(n \geq 2)$ & Cyclic group of order $n$, generated by the $n$-fold rotation $\mathbf{Q}\left(\mathbf{e}_{3}, \theta=2 \pi / n\right)$ \\
\hline$D_{n}(n \geq 2)$ & Dihedral group of order $2 n$ generated by $\mathrm{Z}_{n}$ and $\mathbf{Q}\left(\mathbf{e}_{1}, \pi\right)$ \\
\hline$S O(3)$ & Tridimensional rotational group \\
\hline$O(3)$ & Tridimensional orthogonal group \\
\hline $\mathcal{T}$ & Tetrahedral group of 12 elements generated by $\mathbf{Q}\left(\mathbf{e}_{3}, \pi\right), \mathbf{Q}\left(\mathbf{e}_{1}, \pi\right), \mathbf{Q}(\mathbf{v}, 2 \pi / 3)$ with $\mathbf{v}=\frac{\sqrt{3}}{3}\left(\mathbf{e}_{1}+\mathbf{e}_{2}+\mathbf{e}_{3}\right)$ \\
\hline $\mathcal{O}$ & Octahedral group of 24 elements generated by $\mathbf{Q}\left(\mathbf{e}_{3}, \pi / 2\right), \mathbf{Q}\left(\mathbf{e}_{1}, \pi\right), \mathbf{Q}(\mathbf{v}, 2 \pi / 3)$ with $\mathbf{v}=\frac{\sqrt{3}}{3}\left(\mathbf{e}_{1}+\mathbf{e}_{2}+\mathbf{e}_{3}\right)$ \\
\hline $\mathcal{I}$ & $\begin{array}{l}\text { Dodecahedron group of } 60 \text { elements generated by } \mathbf{Q}\left(\mathbf{e}_{3}, 2 \pi / 5\right), \mathbf{Q}\left(\mathbf{e}_{1}, \pi\right), \mathbf{Q}(\mathbf{w}, 2 \pi / 3) \\
\text { with } \mathbf{w}=\frac{1}{\sqrt{4+\phi^{2}}}\left(2 \mathbf{e}_{2}+\phi \mathbf{e}_{3}\right) \text { and } \phi=\frac{\sqrt{5}+1}{2}\end{array}$ \\
\hline$S O(2)$ & Subgroup of rotations $\mathbf{Q}\left(\mathbf{e}_{3}, \theta\right)$ with $\theta \in[0 ; 2 \pi)$ \\
\hline$O(2)$ & Subgroup generated by $S O(2)$ and $\mathbf{Q}\left(\mathbf{e}_{1}, \pi\right)$ \\
\hline $\mathbf{P}(\mathbf{n})$ & Reflection transformation through the plane of normal $\mathbf{n}$ \\
\hline $\mathbf{P}_{\mathbb{F}}$ & Set of reflection symmetry elements of $\mathbb{F}$ \\
\hline$\left\{\mathbf{P}_{\mathbb{F}}\right\}$ & Reflection symmetry class of $\mathbb{F}$ \\
\hline $\mathcal{P}_{h}$ & Set containing one reflection $\mathbf{P}\left(\mathbf{e}_{3}\right)$ \\
\hline $\mathcal{P}_{v_{k}}(k \geq 1)$ & Set containing $k$ elements $\mathcal{P}_{v_{k}}=\left\{\mathbf{P}\left(\mathbf{r}_{3}\left(\frac{2 p \pi}{k}\right)\right\}_{1 \leq p \leq k}\right.$ with $\mathbf{r}_{3}(\theta)=\sin \theta \mathbf{e}_{1}+\cos \theta \mathbf{e}_{2}$ \\
\hline $\mathcal{P}_{h v_{k}}(k \geq 1)$ & Set containing $k$ elements of $\mathcal{P}_{v_{k}}$ completed by $\mathbf{P}\left(\mathbf{e}_{3}\right)$ \\
\hline $\mathcal{P}_{\mathcal{O}}-1$ & $\begin{array}{l}\text { Cubic set consisting of } 9 \text { reflections with respect to the nine planes of which the normals of } 6 \text { pass through } \\
\text { the center of each edge of a regular cube and the normals of } 3 \text { through the center of each face of the latter }\end{array}$ \\
\hline $\mathcal{P}_{\mathcal{I}}$ & $\begin{array}{l}\text { Icosahedral set of } 15 \text { reflections with respect to the fifteen planes whose normals pass through the center } \\
\text { of each edge of a regular icosahedron }\end{array}$ \\
\hline $\mathcal{P}_{\mathrm{O}(3)}$ & Set composed of all reflections $\mathbf{P}(\mathbf{n})$ \\
\hline
\end{tabular}

With the above notion of conjugacy, a family of nonempty subsets, $\left(\mathcal{F}_{i}\right)_{1 \leq i \leq N}$, of $\mathcal{F}$ acts as a partition of the flexoelectric tensor space $\mathcal{F}$ in the sense that no two elements of $\left(\mathcal{F}_{i}\right)_{1 \leq i \leq N}$ overlap and the union of $\left(\mathcal{F}_{i}\right)_{1 \leq i \leq N}$ is equal to $\mathcal{F}$. Thus, each element $\mathcal{F}_{i}$ of this partition characterizes a symmetry class for flexoelectric tensors.

On the other hand, for a given flexoelectric tensor $\mathbb{F}_{i} \in$ $\mathcal{F}_{i}$ with the symmetry group $\mathcal{G}\left(\mathbb{F}_{i}\right)$, the collection of all the conjugates of $\mathcal{G}\left(\mathbb{F}_{i}\right)$ in the set of subgroups of $S O(3)$, i.e.

$$
\left\{\mathcal{G}_{i}\right\}=\left\{\mathcal{G}\left(\mathbb{F}_{i}\right)\right\}=\left\{\tilde{\mathcal{G}} \subseteq S O(3) \mid \tilde{\mathcal{G}}=\mathbf{Q} \mathcal{G}\left(\mathbb{F}_{i}\right) \mathbf{Q}^{T}, \mathbf{Q} \in S O(3)\right\}
$$

constitutes an intrinsic characterization of the type of rotational symmetries exhibited by the elements of $\mathcal{F}_{i}$. Clearly, the definition of $\mathcal{F}_{i}$ through $\left\{\mathcal{G}_{i}\right\}$ is more convenient. Finally, we denote by $\{G\}$ the collection of all the conjugates of $G \in S O(3)$ in the set of subgroups of $S O(3)$ and define $\mathcal{F}(G)$ as the set

$$
\mathcal{F}(G)=\{\mathbb{F} \in \mathcal{F} \mid \mathcal{G}(\mathbb{F}) \in\{G\}\} .
$$

For the convenience of the reader, the notations used in this paper is summarized in Table 1 . 


\section{ROTATIONAL SYMMETRY CLASSES OF THE FLEXOELECTRIC TENSOR}

For the paper to be self-contained and for later use, the present section recalls the main results of our previous study ${ }^{39}$ concerning the determination of the number and types of all the rotational symmetries for the flexoelectric tensor. First, using a general method due to Spencer $\stackrel{41}{ }$, any fourth-order flexoelectric tensor $\mathbb{F} \in \mathcal{F}$ can be first decomposed into totally symmetric tensors and then split into harmonic tensors. The following explicit harmonic decomposition is established for the flexoelectric tensor $\mathbb{F}$ :

$$
\begin{aligned}
F_{i j k l} & =[\mathbb{H}]_{i j k l} \\
& +\frac{1}{3}\left(3 \epsilon_{i j m}\left[\mathbb{H}^{(1)}\right]_{k l m}+\epsilon_{j k m}\left[\mathbb{H}^{(1)}\right]_{i l m}+\epsilon_{i k m}\left[\mathbb{H}^{(1)}\right]_{j l m}\right)+\frac{1}{4}\left(\epsilon_{i l m}\left[\mathbb{H}^{(2)}\right]_{j k m}+\epsilon_{j l m}\left[\mathbb{H}^{(2)}\right]_{i k m}+\epsilon_{k l m}\left[\mathbb{H}^{(2)}\right]_{i j m}\right) \\
& +\frac{1}{7}\left(\delta_{i j}\left[\mathbf{H}^{(1)}\right]_{k l}+\delta_{i k}\left[\mathbf{H}^{(1)}\right]_{j l}+\delta_{i l}\left[\mathbf{H}^{(1)}\right]_{j k}+\delta_{j k}\left[\mathbf{H}^{(1)}\right]_{i l}+\delta_{j l}\left[\mathbf{H}^{(1)}\right]_{i k}+\delta_{k l}\left[\mathbf{H}^{(1)}\right]_{i j}\right) \\
& +\frac{1}{6}\left(3 \epsilon_{i j m} \epsilon_{k l n}+\epsilon_{i k m} \epsilon_{j l n}+\epsilon_{j k m} \epsilon_{i l n}\right)\left[\mathbf{H}^{(2)}\right]_{m n} \\
& +\frac{2}{9}\left(2 \delta_{j k}\left[\mathbf{H}^{(3)}\right]_{i l}-\delta_{i k}\left[\mathbf{H}^{(3)}\right]_{j l}+\delta_{j l}\left[\mathbf{H}^{(3)}\right]_{i k}-2 \delta_{i l}\left[\mathbf{H}^{(3)}\right]_{j k}+\delta_{l k}\left[\mathbf{H}^{(3)}\right]_{j i}-\delta_{i j}\left[\mathbf{H}^{(3)}\right]_{k l}\right) \\
& +\frac{1}{6}\left(\delta_{j l}\left[\mathbf{H}^{(4)}\right]_{i k}+\delta_{k l}\left[\mathbf{H}^{(4)}\right]_{i j}+\delta_{i l}\left[\mathbf{H}^{(4)}\right]_{j k}-\delta_{i j}\left[\mathbf{H}^{(4)}\right]_{k l}-\delta_{i k}\left[\mathbf{H}^{(4)}\right]_{j l}-\delta_{j k}\left[\mathbf{H}^{(4)}\right]_{i l}\right) \\
& +\frac{3}{10}\left(\delta_{i j} \epsilon_{k l m}+\delta_{j k} \epsilon_{i l m}+\delta_{i k} \epsilon_{j l m}\right)\left[\mathbf{a}^{(1)}\right]_{m} \\
& +\frac{1}{12}\left(2 \epsilon_{k l i}\left[\mathbf{a}^{(2)}\right]_{j}-\epsilon_{k l j}\left[\mathbf{a}^{(2)}\right]_{i}\right)+\frac{1}{12}\left(2 \epsilon_{k i m} \delta_{j l}+2 \epsilon_{l i m} \delta_{j k}-\epsilon_{l j m} \delta_{i k}-\epsilon_{k j m} \delta_{i l}-\epsilon_{l k m} \delta_{i j}\right)\left[\mathbf{a}^{(2)}\right]_{m} \\
& +\frac{1}{15}\left(11 \epsilon_{i k m} \delta_{j l}-4 \epsilon_{j k m} \delta_{i l}-5 \epsilon_{j l m} \delta_{i k}+10 \epsilon_{i l m} \delta_{j k}-5 \epsilon_{k l m} \delta_{i j}+3 \epsilon_{i j m} \delta_{k l}\right)\left[\mathbf{a}^{(3)}\right]_{m} \\
& +\frac{1}{15}\left(3 \epsilon_{i j k}\left[\mathbf{a}^{(3)}\right]_{l}+3 \epsilon_{i j l}\left[\mathbf{a}^{(3)}\right]_{k}+\epsilon_{i k l}\left[\mathbf{a}^{(3)}\right]_{j}+\epsilon_{j k l}\left[\mathbf{a}^{(3)}\right]_{i}\right) \\
& +\frac{\alpha_{1}}{15}\left(\delta_{i j} \delta_{k l}+\delta_{i k} \delta_{j l}+\delta_{i l} \delta_{j k}\right)+\frac{\alpha_{2}}{3}\left(\delta_{j l} \delta_{i k}+\delta_{i j} \delta_{k l}-2 \delta_{i l} \delta_{j k}\right) .
\end{aligned}
$$

It can be seen from (8) that the harmonic decomposition of $\mathbb{F}$ contains : two scalars $\alpha_{1}$ and $\alpha_{2}$; three vectors $\mathbf{a}^{(1)}$, $\mathbf{a}^{(2)}$ and $\mathbf{a}^{(3)}$; four second-order harmonic tensors $\mathbf{H}^{(1)}$, $\mathbf{H}^{(2)}, \mathbf{H}^{(3)}$ and $\mathbf{H}^{(4)}$; two third-order harmonic tensors
$\mathbb{H}^{(1)}$ and $\mathbb{H}^{(2)}$ and a fourth-order harmonic tensor $\mathbb{H}$. The components of these harmonic tensors are explicitly expressed in terms of $F_{i j k l}$ as

$$
\alpha_{1}=\frac{1}{3}\left(F_{p q q p}+2 F_{p p q q}\right), \quad \alpha_{2}=\frac{1}{3} \epsilon_{p q k} \epsilon_{m n k} F_{(m p) n q},
$$

$$
\left[\mathbf{a}^{(1)}\right]_{k}=\frac{1}{9} \epsilon_{p q k}\left(F_{p m m q}+2 F_{m m p q}\right), \quad\left[\mathbf{a}^{(2)}\right]_{k}=\epsilon_{m n p} F_{m n k p}, \quad\left[\mathbf{a}^{(3)}\right]_{k}=\frac{1}{6}\left(\epsilon_{p q k} F_{p q m m}+\epsilon_{p q n} F_{p q k n}\right),
$$

$$
\begin{aligned}
& {\left[\mathbf{H}^{(1)}\right]_{k m}=F_{(p p k m)}-\frac{1}{3} \alpha_{1} \delta_{k m}, \quad\left[\mathbf{H}^{(2)}\right]_{k m}=\frac{1}{2}\left(\epsilon_{p q k} \epsilon_{l n m}+\epsilon_{p q m} \epsilon_{l n k}\right) F_{(l p) n q}-\alpha_{2} \delta_{k m},} \\
& {\left[\mathbf{H}^{(3)}\right]_{k m}=\frac{1}{2} \epsilon_{l n q}\left(\epsilon_{p q m} F_{(l p) n k}+\epsilon_{p q k} F_{(l p) n m}\right), \quad\left[\mathbf{H}^{(4)}\right]_{k m}=\frac{1}{2} \epsilon_{l n q}\left(\epsilon_{p q m} F_{(l p k) n}+\epsilon_{p q k} F_{(l p m) n}\right)}
\end{aligned}
$$




$$
\begin{aligned}
{\left[\mathbb{H}^{(1)}\right]_{k m n} } & =\left[\mathbb{S}^{(1)}\right]_{(k m n)}-\frac{1}{5}\left(\delta_{m n}\left[\mathbf{a}^{(3)}\right]_{k}+\delta_{k m}\left[\mathbf{a}^{(3)}\right]_{n}+\delta_{k n}\left[\mathbf{a}^{(3)}\right]_{m}\right), \\
{\left[\mathbb{H}^{(2)}\right]_{k m n} } & =\left[\mathbb{S}^{(2)}\right]_{(k m n)}-\frac{1}{5}\left(\delta_{m n}\left[\mathbf{a}^{(1)}\right]_{k}+\delta_{k m}\left[\mathbf{a}^{(1)}\right]_{n}+\delta_{k n}\left[\mathbf{a}^{(1)}\right]_{m}\right),
\end{aligned}
$$

$$
\begin{aligned}
{[\mathbb{H}]_{k l m n} } & =F_{(k l m n)}-\frac{1}{7}\left(\delta_{k l}\left[\mathbf{H}^{(1)}\right]_{m n}+\delta_{k m}\left[\mathbf{H}^{(1)}\right]_{l n}+\delta_{k n}\left[\mathbf{H}^{(1)}\right]_{l m}+\delta_{l m}\left[\mathbf{H}^{(1)}\right]_{k n}+\delta_{l n}\left[\mathbf{H}^{(1)}\right]_{k m}+\delta_{m n}\left[\mathbf{H}^{(1)}\right]_{k l}\right) \\
& -\frac{\alpha_{1}}{15}\left(\delta_{k l} \delta_{m n}+\delta_{k m} \delta_{l n}+\delta_{k n} \delta_{l m}\right) .
\end{aligned}
$$

Above, $\delta_{i j}$ and $\epsilon_{i j k}$ are the Kronecker delta and permutation symbol, respectively; either $\bullet_{i_{1} i_{2} \ldots i_{n}}$ or $[\bullet]_{i_{1} i_{2} \ldots i_{n}}$ is the component of an $n$ th-order tensor $\bullet ; \bullet_{\left(i_{1} i_{2} \ldots i_{r}\right) i_{r+1} \ldots i_{n}}$ denotes the average of $r$ ! components obtained by permuting the indices $i_{1}, i_{2}, \ldots, i_{r}$ in all possible ways; the third-order tensors $\mathbb{S}_{1}$ and $\mathbb{S}_{2}$ are defined as

$$
\left[\mathbb{S}^{(1)}\right]_{k m n}=\epsilon_{p q n} F_{(p k) q m}, \quad\left[\mathbb{S}^{(2)}\right]_{k m n}=\epsilon_{p q n} F_{(p k m) q} .
$$

Next, by using the Cartan method, the second-, thirdand fourth-order harmonic tensors $\mathbf{H}^{(i)}, \mathbb{H}^{(i)}$ and $\mathbb{H}$ in Eq. (8) can be rewritten as follows:

$$
\begin{aligned}
& \mathbf{H}^{(i)}=\alpha_{0 i}^{(2)} \mathbf{U}_{0}+\alpha_{1 i}^{(2)} \mathbf{U}_{1}+\beta_{1 i}^{(2)} \mathbf{T}_{1}+\alpha_{2 i}^{(2)} \mathbf{U}_{2}+\beta_{2 i}^{(2)} \mathbf{T}_{2} \text { with } i=1,2,3,4, \\
& \mathbb{H}^{(i)}=\alpha_{0 i}^{(3)} \mathbb{U}_{0}+\alpha_{1 i}^{(3)} \mathbb{U}_{1}+\beta_{1 i}^{(3)} \mathbb{T}_{1}+\alpha_{2 i}^{(3)} \mathbb{U}_{2}+\beta_{2 i}^{(3)} \mathbb{T}_{2}+\alpha_{3 i}^{(3)} \mathbb{U}_{3}+\beta_{3 i}^{(3)} \mathbb{T}_{3} \text { with } i=1,2, \\
& \mathbb{H}=\alpha_{0}^{(4)} \mathbb{U}_{0}+\alpha_{1}^{(4)} \mathbb{U}_{1}+\beta_{1}^{(4)} \mathbb{T}_{1}+\alpha_{2}^{(4)} \mathbb{U}_{2}+\beta_{2}^{(4)} \mathbb{T}_{2}+\alpha_{3}^{(4)} \mathbb{U}_{3}+\beta_{3}^{(4)} \mathbb{T}_{3}+\alpha_{4}^{(4)} \mathbb{U}_{4}+\beta_{4}^{(4)} \mathbb{T}_{4},
\end{aligned}
$$

where $\mathbf{U}_{i}, \mathbf{T}_{i}, \mathbb{U}_{i}, \mathbb{T}_{i}, \mathbb{U}_{i}$ and $\mathbb{T}_{i}$ are the tensors involved in the Cartan decomposition, whose explicit expressions can be found in Forte and Vianelld ${ }^{42 / 43}$ or Le Quang and $\mathrm{He}^{39}$.

With the help of the harmonic and Cartan decompositions presented above, it can be shown that the number of all possible rotational symmetry classes for all flexoelectric tensors is 12 . These 12 symmetry classes are characterized by the conjugates of the following 12 sub- groups of $S O(3)$ :

$$
I,\left\{Z_{r}\right\},\left\{D_{r}\right\},\{\mathcal{O}\},\{\mathcal{T}\},\{S O(2)\},\{O(2)\}, S O(3)
$$

where $2 \leq r \leq 4$.

Alternatively, the 12 sets $\mathcal{F}(I), \mathcal{F}\left(Z_{r}\right), \mathcal{F}\left(D_{r}\right)$ with $2 \leq r \leq 4, \mathcal{F}(\mathcal{O}), \mathcal{F}(\mathcal{T}), \mathcal{F}(S O(2)), \mathcal{F}(O(2)), \mathcal{F}(S O(3))$ form a partition of the flexoelectric tensor space $\mathcal{F}$ :

$$
\mathcal{F}=\mathcal{F}(I) \cup_{r=2}^{4} \mathcal{F}\left(Z_{r}\right) \cup_{r=2}^{4} \mathcal{F}\left(D_{r}\right) \cup \mathcal{F}(\mathcal{O}) \cup \mathcal{F}(\mathcal{T}) \cup \mathcal{F}(S O(2)) \cup \mathcal{F}(O(2)) \cup \mathcal{F}(S O(3))
$$

For more detail about the derivation of these results, the reader can refer to our previous work ${ }^{39}$.

\section{COMPACT EXPLICIT MATRIX REPRESENTATIONS OF THE FLEXOELECTRIC TENSOR}

In order to obtain explicit matrix expressions of the fourth-order flexoelectric tensor for all the 12 rotational symmetry classes, we first adopt the following reduced suffix notations for the gradient $\mathbb{E}$ of the infinitesimal strain tensor $\varepsilon$ and for the fourth-order flexoelectric tensor $\mathbb{F} \in \mathcal{F}$ :

$$
\tilde{E}_{\gamma}=\left[\sqrt{2}\left(1-\delta_{j k}\right)+\delta_{j k}\right] E_{j k l},
$$

$$
\tilde{F}_{i \gamma}=\left[\sqrt{2}\left(1-\delta_{j k}\right)+\delta_{j k}\right] F_{i j k l}
$$


TABLE II. Suffix notation correspondences between $(j, k, l)$ and $\gamma$.

\begin{tabular}{cc}
\hline \hline$(j, k, l)$ or $(m, n, s)$ & $\gamma$ or $\zeta$ \\
\hline$(1,1,1)$ & 1 \\
$(2,2,1)$ & 2 \\
$(1,2,2)$ or $(2,1,2)$ & 3 \\
$(3,3,1)$ & 4 \\
$(1,3,3)$ or $(3,1,3)$ & 5 \\
$(2,2,2)$ & 6 \\
$(1,1,2)$ & 7 \\
$(1,2,1)$ or $(2,1,1)$ & 8 \\
$(3,3,2)$ & 9 \\
$(2,3,3)$ or $(3,2,3)$ & 10 \\
$(3,3,3)$ & 11 \\
$(1,1,3)$ & 12 \\
$(1,3,1)$ or $(3,1,1)$ & 13 \\
$(2,2,3)$ & 14 \\
$(2,3,2)$ or $(3,2,2)$ & 15 \\
$(1,2,3)$ or $(2,1,3)$ & 16 \\
$(1,3,2)$ or $(3,1,2)$ & 17 \\
$(2,3,1)$ or $(3,2,1)$ & 18 \\
\hline \hline
\end{tabular}

where $\gamma$ defined in Table $\Pi$ and the summation convention does not apply on $j$ and $k$.

According to the previously reduced notation rules, the constitutive relation (3) between the electric polarization and the gradient of the infinitesimal strain tensor can now be written in the following matrix form:

$$
\mathbf{p}=\tilde{\mathbf{F}} \cdot \tilde{\mathbf{E}}
$$

where the vectors $\mathbf{p}$ and $\tilde{\mathbf{E}}$ are specified by

$$
\begin{aligned}
& \mathbf{p}=\left[\begin{array}{lll}
p_{1} & p_{2} & p_{3}
\end{array}\right]^{T}, \\
& \tilde{\mathbf{E}}=\left[\begin{array}{llll}
\tilde{E}_{1} & \tilde{E}_{2} & \ldots & \tilde{E}_{18}
\end{array}\right]^{T},
\end{aligned}
$$

and $\tilde{\mathbf{F}}$ is the $3 \times 18$ flexoelectric matrix whose block matrix representation has the general expression

$$
\tilde{\mathbf{F}}=\left[\begin{array}{cccc}
D_{x} & C_{x y} & C_{x z} & J_{x} \\
C_{y x} & D_{y} & C_{y z} & J_{y} \\
C_{z x} & C_{z y} & D_{z} & J_{z}
\end{array}\right] .
$$

Here, the diagonal block matrices $D_{x}, D_{y}$ and $D_{z}$ of $D$ type and extra-diagonal block matrices $C_{x y}, C_{x z}, C_{y x}$, $C_{y z} C_{z x}$ and $C_{z y}$ of $C$-type have the same size $1 \times 5$ while the extra-diagonal block matrices $J_{x}, J_{y}$ and $J_{z}$ of $J$-type are of the size $1 \times 3$.

With the previously reduced notation rules, the action of an orthogonal tensor $\mathbf{Q} \in O(3)$ on a flexoelectric tensor $\mathbb{F}$ can be expressed in the following simple and explicit matrix form:

$$
Q_{i r} Q_{j m} Q_{k n} Q_{l s} F_{r m n s}=Q_{i r} \tilde{F}_{r \zeta} \tilde{Q}_{\zeta \gamma}
$$

where $\tilde{Q}_{\zeta \gamma}$, standing for the components of the $18 \times 18$ matrix $\tilde{\mathbf{Q}}$, are given by

$$
\begin{aligned}
\tilde{Q}_{\zeta \gamma} & =\left[\sqrt{2}\left(1-\delta_{j k}\right)+\delta_{j k}\right] \\
& \times\left[\sqrt{2}\left(1-\delta_{m n}\right)+\delta_{m n}\right] Q_{j m} Q_{k n} Q_{l s} .
\end{aligned}
$$

Above, $\gamma$ and $\zeta$ are defined in Table $\Pi$ and the summation convention does not hold for $j, k, m$ and $n$.

By using the procedure elaborated in Le Quang and $\mathrm{He}^{39}$ to construct a flexoelectric tensor $\mathbb{F}$ exhibiting a required symmetry and by exploiting the harmonic and Cartan decompositions in Eqs. (8) and (15) together with the Cartan decomposition parameters $\alpha_{\bullet}^{(\bullet)}$ and $\beta_{\bullet}^{(\bullet)}$ provided in Table III, we can exactly calculate the number of independent components contained in a flexoelectric tensor $\mathbb{F} \in \mathcal{F}$ belonging to a given symmetry class. The corresponding results are shown in Table IV]

Next, by adopting the above reduced suffix notations for the fourth-order flexoelectric tensor $\mathbb{F}$, we obtain the explicit matrix forms of the elementary block matrices of $(D, C, J)$-type as well as the explicit matrix form of $\tilde{\mathbf{F}}$ for each symmetry class. These explicit block matrix forms of $\tilde{\mathbf{F}}$ are provided as follows:

Identity class: $I$

$$
\tilde{\mathbf{F}}_{I}=\left[\begin{array}{cccc}
D_{x}^{(5)} & C_{x y}^{(5)} & C_{x z}^{(5)} & J_{x}^{(3)} \\
C_{y x}^{(5)} & D_{y}^{(5)} & C_{y z}^{(5)} & J_{y}^{(3)} \\
C_{z x}^{(5)} & C_{z y}^{(5)} & D_{z}^{(5)} & J_{z}^{(3)}
\end{array}\right],
$$

Cyclic classes: $Z_{r}$

$$
\begin{aligned}
\tilde{\mathbf{F}}_{Z_{2}}= & {\left[\begin{array}{cccc}
D_{x}^{(5)} & C_{x y}^{(5)} & 0 & 0 \\
C_{y x}^{(5)} & D_{y}^{(5)} & 0 & 0 \\
0 & 0 & D_{z}^{(5)} & J_{z}^{(3)}
\end{array}\right], } \\
\tilde{\mathbf{F}}_{Z_{3}}= & {\left[\begin{array}{cccc}
D^{(4)} & C_{x y}^{(4)} & C_{x z}^{(2)} & C_{y z}^{(2)} \cdot P_{1} \\
-C_{x y}^{(4)} & D^{(4)} & C_{y z}^{(2)} & -C_{x z}^{(2)} \cdot P_{1} \\
C_{z x}^{(1)} & C_{z y}^{(1)} & D_{z}^{(3)} & J_{z}^{(1)}
\end{array}\right], } \\
\tilde{\mathbf{F}}_{Z_{4}}= & {\left[\begin{array}{cccc}
D^{(5)} & C_{x y}^{(5)} & 0 & 0 \\
-C_{x y}^{(5)} & D^{(5)} & 0 & 0 \\
0 & 0 & D_{z}^{(3)} & J_{z}^{(1)}
\end{array}\right] } \\
\tilde{\mathbf{F}}_{S O(2)}= & {\left[\begin{array}{cccc}
D^{(4)} & C_{x y}^{(4)} & 0 & 0 \\
-C_{x y}^{(4)} & D^{(4)} & 0 & 0 \\
0 & 0 & D_{z}^{(3)} & J_{z}^{(1)}
\end{array}\right], }
\end{aligned}
$$

Dihedral classes: $D_{r}$

$$
\begin{aligned}
\tilde{\mathbf{F}}_{D_{2}}= & {\left[\begin{array}{cccc}
D_{x}^{(5)} & 0 & 0 & 0 \\
0 & D_{y}^{(5)} & 0 & 0 \\
0 & 0 & D_{z}^{(5)} & 0
\end{array}\right], } \\
\tilde{\mathbf{F}}_{D_{3}}= & {\left[\begin{array}{cccc}
D^{(4)} & 0 & 0 & C_{y z}^{(2)} \cdot P_{1} \\
0 & D^{(4)} & C_{y z}^{(2)} & 0 \\
0 & C_{z y}^{(1)} & D_{z}^{(3)} & 0
\end{array}\right], } \\
\tilde{\mathbf{F}}_{D_{4}}= & {\left[\begin{array}{cccc}
D^{(5)} & 0 & 0 & 0 \\
0 & D^{(5)} & 0 & 0 \\
0 & 0 & D_{z}^{(3)} & 0
\end{array}\right] } \\
\tilde{\mathbf{F}}_{O(2)}= & {\left[\begin{array}{cccc}
D^{(4)} & 0 & 0 & 0 \\
0 & D^{(4)} & 0 & 0 \\
0 & 0 & D_{z}^{(3)} & 0
\end{array}\right], }
\end{aligned}
$$


TABLE III. Zero components · and non-zero independent material parameters • contained in a flexoelectric tensor belonging to a given symmetry class

\begin{tabular}{|c|c|c|c|c|c|c|c|c|c|c|c|c|}
\hline Parameters & $I$ & $\left\{Z_{2}\right\}$ & $\left\{D_{2}\right\}$ & $\left\{Z_{3}\right\}$ & $\left\{D_{3}\right\}$ & $\left\{Z_{4}\right\}$ & $\left\{D_{4}\right\}$ & $\{\mathcal{T}\}^{a}$ & $\{\mathcal{O}\}^{b}$ & $\{O(2)\}$ & $\{S O(2)\}$ & $\{S O(3)\}$ \\
\hline$\alpha_{1}$ & $\bullet$ & $\bullet$ & $\bullet$ & $\bullet$ & $\bullet$ & $\bullet$ & $\bullet$ & $\bullet$ & $\bullet$ & $\bullet$ & $\bullet$ & $\bullet$ \\
\hline$\alpha_{2}$ & • & • & • & • & • & • & • & • & • & • & • & • \\
\hline$a_{1}^{(i)}$ & $\bullet$ & . & . & . & . & . & . & . & . & . & . & . \\
\hline$a_{2}^{(i)}$ & $\bullet$ & . & . & . & . & . & . & . & . & . & . & . \\
\hline$a_{3}^{(i)}$ & - & . & - & - & . & - & . & . & . & . & - & . \\
\hline$\alpha_{0 i}^{(2)}$ & $\bullet$ & $\bullet$ & $\bullet$ & $\bullet$ & $\bullet$ & • & • & . & . & • & • & . \\
\hline$\alpha_{1 i}^{(2)}$ & $\bullet$ & & . & . & . & 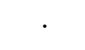 & . & . & . & . & . & . \\
\hline$\beta_{1 i}^{(1)}$ & • & . & . & . & . & . & . & . & . & . & . & . \\
\hline$\alpha_{2 i}^{(2)}$ & $\bullet$ & $\bullet$ & $\bullet$ & . & . & . & . & . & . & . & . & . \\
\hline$\beta_{2 i}^{(i)}$ & • & • & . & . & . & . & . & . & . & . & . & . \\
\hline$\alpha_{0 i}^{(3)}$ & • & • & . & • & . & • & . & . & . & . & • & . \\
\hline$\alpha_{1 i}^{(3)}$ & • & . & . & . & . & . & . & . & . & . & . & . \\
\hline$\beta_{1 i}^{(3)}$ & • & . & . & . & . & & . & . & & . & . & . \\
\hline$\alpha_{2 i}^{(3)}$ & • & • & . & . & . & 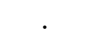 & . & . & . & . & . & . \\
\hline$\beta_{2 i}^{(3)}$ & • & • & • & . & . & . & . & • & . & . & . & . \\
\hline$\alpha_{3 i}^{(3)}$ & • & . & . & $\bullet$ & $\bullet$ & . & . & . & . & . & . & . \\
\hline$\beta_{3 i}^{(3)}$ & • & . & . & • & . & . & . & . & . & . & . & . \\
\hline$\alpha_{0}^{(4)}$ & - & • & $\bullet$ & $\bullet$ & $\bullet$ & $\bullet$ & $\bullet$ & $\bullet$ & • & $\bullet$ & $\bullet$ & . \\
\hline$\alpha_{1}^{(4)}$ & • & & . & . & . & 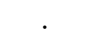 & . & . & & . & . & . \\
\hline$\beta_{1}^{(4)}$ & - & . & . & . & . & . & . & . & & . & . & . \\
\hline$\alpha_{2}^{(4)}$ & $\bullet$ & $\bullet$ & $\bullet$ & . & . & . & . & . & . & . & . & . \\
\hline$\beta_{2}^{(4)}$ & $\bullet$ & $\bullet$ & . & . & . & . & . & . & . & . & . & . \\
\hline$\alpha_{3}^{(4)}$ & • & . & . & $\bullet$ & . & . & . & . & . & . & . & . \\
\hline$\beta_{3}^{(4)}$ & - & . & . & $\bullet$ & • & . & . & . & . & . & . & . \\
\hline$\alpha_{4}^{(4)}$ & • & • & • & . & . & • & • & • & • & . & . & . \\
\hline$\beta_{4}^{(4)}$ & • & • & . & . & r. & • & . & 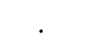 & & . & . & r. \\
\hline
\end{tabular}

a $\alpha_{4}^{(4)}=5 \alpha_{0}^{(4)}$

b $\alpha_{4}^{(4)}=5 \alpha_{0}^{(4)}$

TABLE IV. Number of independent material parameters contained in a flexoelectric tensor belonging to a given symmetry class

\begin{tabular}{|c|c|c|c|c|c|c|c|c|c|c|c|c|}
\hline Symmetry class & $I$ & $\left\{Z_{2}\right\}$ & $\left\{D_{2}\right\}$ & $\left\{Z_{3}\right\}$ & $\left\{D_{3}\right\}$ & $\left\{Z_{4}\right\}$ & $\left\{D_{4}\right\}$ & $\{\mathcal{T}\}$ & $\{\mathcal{O}\}$ & $\{O(2)\}$ & $\{S O(2)\}$ & $\{S O(3)\}$ \\
\hline Number of independent components & 54 & 28 & 15 & 18 & 10 & 14 & 8 & 5 & 3 & 7 & 12 & 2 \\
\hline
\end{tabular}

\section{Spatial classes:}

$$
\begin{aligned}
& \tilde{\mathbf{F}}_{\mathcal{T}}=\left[\begin{array}{cccc}
D^{(5)} & 0 & 0 & 0 \\
0 & D^{(5)} \cdot P_{2} & 0 & 0 \\
0 & 0 & D^{(5)} & 0
\end{array}\right], \\
& \tilde{\mathbf{F}}_{\mathcal{O}}=\left[\begin{array}{cccc}
D^{(3)} & 0 & 0 & 0 \\
0 & D^{(3)} & 0 & 0 \\
0 & 0 & D^{(3)} & 0
\end{array}\right], \\
& \tilde{\mathbf{F}}_{S O(3)}=\left[\begin{array}{cccc}
D^{(2)} & 0 & 0 & 0 \\
0 & D^{(2)} & 0 & 0 \\
0 & 0 & D^{(2)} & 0
\end{array}\right] .
\end{aligned}
$$

In Eqs. (25)-(35), the superscript of each elementary block matrix of $D, C$ or $J$-type denotes the number of independent material parameters; $P_{1}$ and $P_{2}$ are two cor- rection matrices. By introducing two material parameters defined as

$$
\eta=\frac{1}{\sqrt{2}}\left(\tilde{F}_{11}-\tilde{F}_{12}\right), \quad \theta=\frac{1}{\sqrt{2}}\left(\tilde{F}_{22}-\tilde{F}_{21}\right)
$$

the elementary block matrices of $(D, C, J)$-types as well as of the correction matrices $P_{1}$ and $P_{2}$ are explicitly expressed as follows:

\section{D-type elements:}

$$
\begin{aligned}
& D^{(5)}=\left[\begin{array}{lllll}
d_{1} & d_{2} & d_{3} & d_{4} & d_{5}
\end{array}\right], \\
& D^{(4)}=\left[\begin{array}{lllll}
d_{1} & d_{2} & \eta & d_{4} & d_{5}
\end{array}\right], \\
& D^{(3)}=\left[\begin{array}{lllll}
d_{1} & d_{2} & d_{3} & d_{2} & d_{3}
\end{array}\right], \\
& D^{(2)}=\left[\begin{array}{lllll}
d_{1} & d_{2} & \eta & d_{2} & \eta
\end{array}\right] \text {, }
\end{aligned}
$$




\section{C-type elements:}

$$
\begin{aligned}
& C^{(5)}=\left[\begin{array}{lllll}
c_{1} & c_{2} & c_{3} & c_{4} & c_{5}
\end{array}\right], \\
& C^{(4)}=\left[\begin{array}{lllll}
c_{1} & c_{2} & \theta & c_{4} & c_{5}
\end{array}\right], \\
& C^{(2)}=\left[\begin{array}{lllll}
0 & c_{2} & c_{3} & -c_{2} & -c_{3}
\end{array}\right], \\
& C^{(1)}=\left[\begin{array}{lllll}
c_{1} & -c_{1} & -c_{1} & 0 & 0
\end{array}\right],
\end{aligned}
$$

J-type elements:

$$
J^{(3)}=\left[\begin{array}{lll}
j_{1} & j_{2} & j_{3}
\end{array}\right], \quad J^{(1)}=\left[\begin{array}{lll}
0 & j_{2} & -j_{2}
\end{array}\right],
$$

Correction elements:

$$
P_{1}=\left[\begin{array}{lll}
0 & 0 & 0 \\
1 & 0 & 0 \\
0 & 1 & 1 \\
0 & 0 & 0 \\
0 & 0 & 1
\end{array}\right], P_{2}=\left[\begin{array}{lllll}
1 & 0 & 0 & 0 & 0 \\
0 & 0 & 0 & 1 & 0 \\
0 & 0 & 0 & 0 & 1 \\
0 & 1 & 0 & 0 & 0 \\
0 & 0 & 1 & 0 & 0
\end{array}\right] .
$$

Finally, the compact explicit matrix expressions for the 12 symmetry classes of flexoelectric tensors are shown in Table V

\section{REFLECTION SYMMETRY CLASSES OF THE FLEXOELECTRIC TENSOR}

First, let us introduce $\mathbf{P}(\mathbf{n}) \in \mathrm{O}(3) \backslash \mathrm{SO}(3)$, the reflection through the plane $\mathcal{P}(\mathbf{n})=\left\{\mathbf{x} \in \mathbb{R}^{3} \mid \mathbf{x} \cdot \mathbf{n}=0\right\}$ perpendicular to a unit vector $\mathbf{n}$, by

$$
\mathbf{P}(\mathbf{n})=\mathbf{I}-2 \mathbf{n} \otimes \mathbf{n} .
$$

It can be seen from (47) that $\mathbf{P}$ is an even function of $\mathbf{n}$ in the sense that $\mathbf{P}(-\mathbf{n})=\mathbf{P}(\mathbf{n})$. Thus, two unit normal vectors are associated to each reflection.

Next, we denote by $\mathbf{P}_{\mathbb{F}}$ the set of reflection symmetry elements of $\mathbb{F} \in \mathcal{F}$. The reflection symmetry class of $\mathbb{F} \in \mathcal{F}$, symbolized by $\left\{\mathbf{P}_{\mathbb{F}}\right\}$, is defined as the collection of all the conjugates of $\mathbf{P}_{\mathbb{F}}$, namely

$$
\left\{\mathbf{P}_{\mathbb{F}}\right\}=\left\{\mathbf{P}_{\mathbb{F}}^{\prime}=\mathbf{R} \mathbf{P}_{\mathbb{F}} \mathbf{R}^{T} \mid \mathbf{R} \in \mathrm{SO}(3)\right\} .
$$

Note that $\left\{\mathbf{P}_{\mathbb{F}}\right\}$ represents the reflection symmetry class and not the symmetry group of $\mathbb{F} \in \mathcal{F}$.

We introduce the unit vector

$$
\mathbf{r}_{i}(\theta)=\sin \theta \mathbf{e}_{j}+\cos \theta \mathbf{e}_{k}
$$

with $\{i, j, k\}$ being a cycle permutation of $\{1,2,3\}$, and the following sets of reflection transformations:

- $\mathcal{P}_{h}$ being the set which contains only the reflection $\mathbf{P}\left(\mathbf{e}_{3}\right)$. The label $h$ means "horizontal".

- $\mathcal{P}_{v_{k}}$ being the set defined by $\mathcal{P}_{v_{k}}=$ $\left\{\mathbf{P}\left(\mathbf{r}_{3}\left(\frac{2 p \pi}{k}\right)\right\}_{1 \leq p \leq k}\right.$ and consisting of $k$ elements. The label $v$ means "vertical".

- $\mathcal{P}_{h v_{k}}(k \geq 1)$ being the set defined by the $k$ elements of $\mathcal{P}_{v_{k}}$ completed by $\mathbf{P}\left(\mathbf{e}_{3}\right)$ : this set comprises $k+1$ elements.
- $\mathcal{P}_{\mathcal{O}}$ designating the cubic set of nine reflections with respect to the nine planes of which the normals of 6 pass through the center of each edge of a regular cube and the normals of 3 through the center of each face of the latter.

- $\mathcal{P}_{\mathcal{I}}$ denoting the icosahedral set consisting of fifteen reflections with respect to the fifteen planes whose normals pass through the center of each edge of a regular icosahedron;

- $\mathcal{P}_{\mathrm{O}(3)}$ representing the set composed of all reflections $\mathbf{P}(\mathbf{n})$ with $\mathbf{n} \in \mathcal{S}^{2}$ where $\mathcal{S}^{2}$ is the unit sphere defined by $\mathcal{S}^{2}=\left\{\mathbf{x} \in \mathbb{R}^{3} \mid\|x\|=1\right\}$.

We can show that the space $\mathcal{F}$ of flexoelectric tensors is divided into the 8 reflection symmetry classes which are characterized by the following 8 sets of reflection transformations:

$$
\emptyset,\left\{\mathcal{P}_{h}\right\},\left\{\mathcal{P}_{h v_{2}}\right\},\left\{\mathcal{P}_{v_{3}}\right\},\left\{\mathcal{P}_{h v_{4}}\right\},\left\{\mathcal{P}_{h v_{\infty}}\right\},\left\{\mathcal{P}_{\mathcal{O}}\right\},\left\{\mathcal{P}_{\mathrm{O}(3)}\right\}
$$

The characteristics of each reflection symmetry class, and especially its link with the rotational ones, are detailed in Table VI] It is important and interesting to remark that: (i) unlike the results as obtained by Chadwick et al. ${ }^{44}$ for the space of fourth-order elasticity tensors, according to which the classifications by rotational symmetry groups and by reflection symmetry planes give the same response, the number of rotational symmetry classes for the space of fourth-order flexoelectric tensors is 12 while the one of reflection symmetry classes is only 8; (ii) these 8 reflection symmetry classes for the space of flexoelectric tensors are exactly identical to the ones for the space of elasticity tensors, even through a fourth-order flexoelectric tensor is algebraically more complex than a fourthorder elasticity tensor.

\section{A GRAPHIC METHOD FOR IDENTIFYING REFLECTION AND ROTATIONAL SYMMETRIES}

The important question now arises as to how to identify the reflection symmetry and rotational symmetry and of a given flexoelectric tensor from the knowledge of its matrix relative to a basis. The present section aims at elaborating a simple but efficient graphic method to answer this question.

Francois et al .45 initiated a graphic approach to identifying the reflection symmetry planes that a given fourthorder elastic tensor has. This approach is based on the notion of "pole figures". Owing to the fact that the reflection symmetry classes of the fourth-order elastic tensor are identical to its rotational symmetry classes, the identification of the formers leads also the one of the latters. However, when the fourth-order elastic tensor is concerned, the situation is much more complicated, since its reflection symmetry classes are 8 while its reflection symmetry classes are 12. Thus, in this section we extend 
TABLE V. Number of independent material parameters and compact explicit matrix expression for each of the 12 symmetry classes of flexoelectric tensors

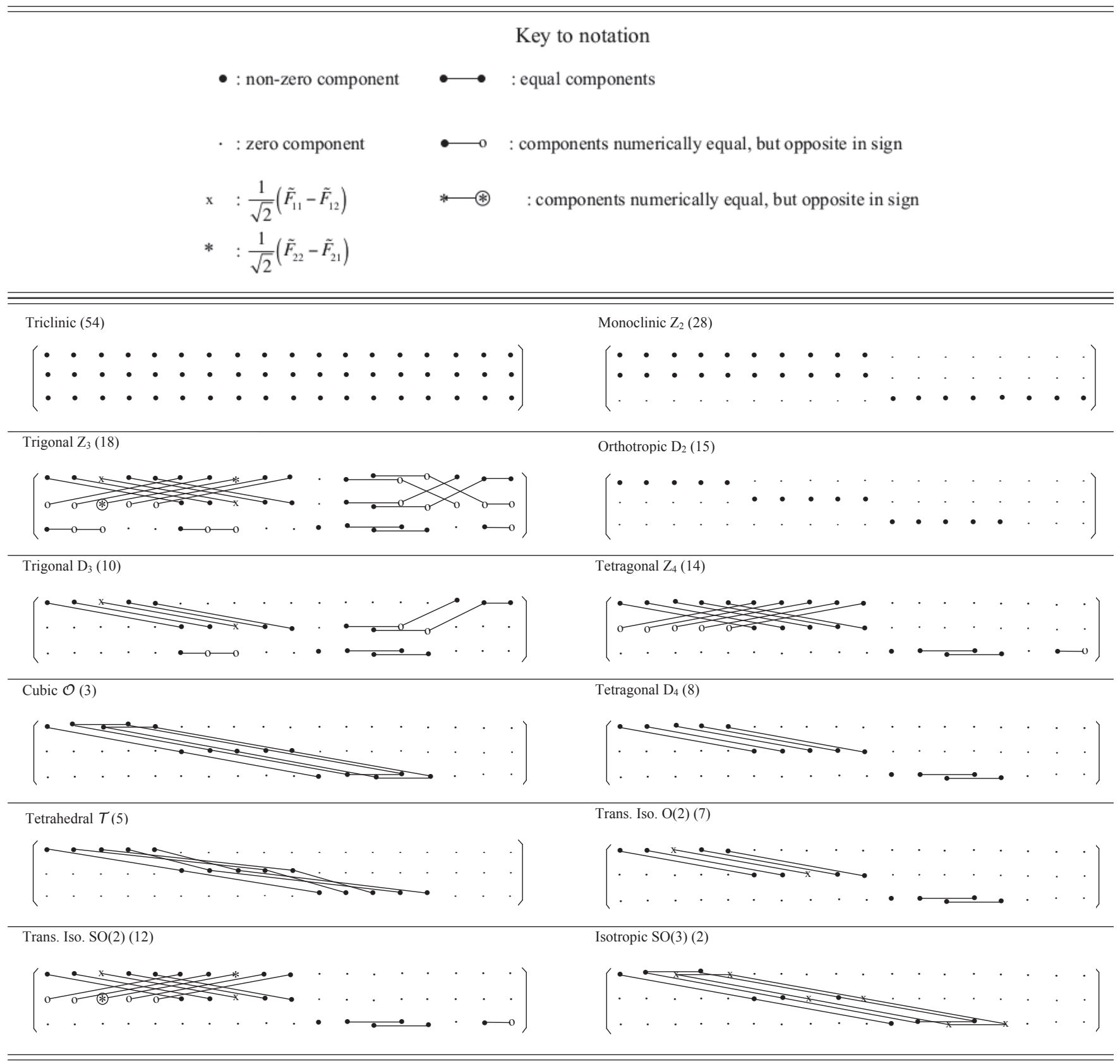

TABLE VI. Symmetry plane stratification of the space $\mathcal{F}$ of fourth-order flexoelectric tensors

\begin{tabular}{|c|c|c|c|}
\hline System & $\begin{array}{c}\text { Reflection } \\
\text { symmetry class }\end{array}$ & $\begin{array}{c}\text { Number of reflection } \\
\text { symmetry planes }\end{array}$ & $\begin{array}{c}\text { Rotational } \\
\text { symmetry class }\end{array}$ \\
\hline \hline Triclinic & $\emptyset$. & 0 & $\mathbf{I},\left\{Z_{3}\right\}$ \\
Monoclinic & $\left\{\mathcal{P}_{h}\right\}$ & 1 & $\left\{Z_{2}\right\},\left\{Z_{4}\right\},\{S O(2)\}$ \\
Orthotropic & $\left\{\mathcal{P}_{h v_{2}}\right\}$ & 3 & $\left\{D_{2}\right\},\{\mathcal{T}\}$ \\
Trigonal & $\left\{\mathcal{P}_{v_{3}}\right\}$ & 3 & $\left\{D_{3}\right\}$ \\
Tetragonal & $\left\{\mathcal{P}_{h v_{4}}\right\}$ & 5 & $\left\{D_{4}\right\}$ \\
Trans. isotropic & $\left\{\mathcal{P}_{h v_{\infty}}\right\}$ & $\infty+1$ & $\{O(2)\}$ \\
Cubic & $\left\{\mathcal{P}_{\mathcal{O}}\right\}$ & 9 & $\{\mathcal{O}\}$ \\
Isotropic & $\left\{\mathcal{P}_{O(3)}\right\}$ & $\infty^{3}$ & $\{S O(3)\}$ \\
\hline
\end{tabular}


the graphic approach of Francois et al $!^{45}$ to being able to identify not only the reflection symmetry of a given flexoelectric tensor but also its rotational symmetry.

Let $\mathbf{n} \in S^{2}$ be the unit vector relative to the reflection transformation $\mathbf{P}(\mathbf{n})=\mathbf{I}-2 \mathbf{n} \otimes \mathbf{n}$ through the plane $\mathcal{P}(\mathbf{n})$. With no loss of generality, $\mathbf{n}$ is expressed by

$$
\mathbf{n}=\sin \theta \cos \phi \mathbf{e}_{1}+\sin \theta \sin \phi \mathbf{e}_{2}+\cos \theta \mathbf{e}_{3}
$$

where $(\phi, \theta) \in[0,2 \pi[\times[0, \pi[$ denote, respectively, the longitude and colatitude angles relative to a system of spherical coordinates. Then, by considering, for a given flexoelectric tensor $\mathbb{F} \in \mathcal{F}$, the function:

$$
L(\theta, \phi)=\|\mathbf{P}(\theta, \phi) * \mathbb{F}-\mathbb{F}\|=\|\mathbf{P}(\theta, \phi) \cdot \tilde{\mathbf{F}} \cdot \tilde{\mathbf{P}}(\theta, \phi)-\tilde{\mathbf{F}}\|
$$

in which $\|\cdot\|$ is the Frobenius norm inherited from the scalar product on $\mathcal{F}, \mathbf{P}(\theta, \phi)$ is a reflection operator parametrized with the longitude and colatitude angles and $\tilde{\mathbf{P}}(\theta, \phi)$ is a $18 \times 18$ matrix whose components are obtained by replacing $\mathbf{Q}$ with $\mathbf{P}$ in 23 . Finally, the vanishing loci of $L(\theta, \phi)$ give the unit normals to the symmetry planes that $\mathbb{F}$ has.

Concretely, the function (52) is numerically evaluated in a discrete way. Precisely, we introduce

$$
M_{i j}=L\left(\theta_{i}, \phi_{j}\right) \quad \text { with } \quad \theta_{i}=i \frac{2 \pi}{N} \quad \text { and } \quad \phi_{j}=j \frac{2 \pi}{N}
$$

where the number $N$ depends on the degree of numerical accuracy required. In our computations, $N$ is set to be equal to 160 . To evaluate the function $L(\theta, \phi)$, we first use the matrix representations of flexoelectric tensors presented in Section IV. In addition, the numerical values of the components of a flexoelectric tensor are determined as random integers picked-up in the range $\{-10,10\}$. We show, in Figure 1, the loci of the zeros of $L(\theta, \phi)$ plotted on the $\theta-\phi$ plane for all rotation symmetry classes and all reflection symmetry classes. It can be seen from Figure 1 that the number of symmetry planes for a given rotation symmetry class or reflection symmetry class coincides exactly with the one provided in Table VI. This also constitutes a validity verification of our theoretical results.

In addition, it can be observed from Figure 1 that, even through the flexoelectric tensors belonging to both rotational symmetry classes $\left\{Z_{3}\right\}$ and $\mathbf{I}$ do not exhibit any reflection symmetry plane, we can differentiate them since the loci of the function $L(\theta, \phi)$ for the flexoelectric tensors belonging to $\left\{Z_{3}\right\}$ are periodic in the $\phi$-direction with period $2 \pi / 3$ while the ones for the flexoelectric tensors appertaining to $\mathbf{I}$ are not periodic. Similarly, even if the flexoelectric tensors belonging to the rotational symmetry classes $\left\{D_{2}\right\}$ and $\mathcal{T}$ possess the same number of reflection symmetry planes, the loci of the function $L(\theta, \phi)$ for flexoelectric tensors belonging to $\{\mathcal{T}\}$ are periodic in the $\phi$-direction with period $\pi / 2$ but the counterpart of the flexoelectric tensors appertaining to $\left\{D_{2}\right\}$ are periodic in $\phi$-direction with period $\pi$.
The foregoing graphical approach is now applied to a given flexoelectric tensor $\mathbb{F} \in \mathcal{F}$ with a given angle $\psi \in[0 ; 2 \pi$ [ for identifying all invariant directions defined by $\mathbf{n}=\sin \theta \cos \phi \mathbf{e}_{1}+\sin \theta \sin \phi \mathbf{e}_{2}+\cos \theta \mathbf{e}_{3}$ in the sense that $\mathbb{F}$ is unchangeable under the rotational transformation action $\mathbf{Q}(\mathbf{n}, \psi)$. As before, by using the well-known Rodrigues expression of $\mathbf{Q}(\mathbf{n}, \psi)$, i.e.

$$
\mathbf{Q}(\mathbf{n}, \psi)=\cos (\psi) \mathbf{I}-\sin (\psi) \epsilon \cdot \mathbf{n}+[1-\cos (\psi)] \mathbf{n} \otimes \mathbf{n}
$$

in which $\epsilon$ denotes the Levi-Civita third-order tensor, and by introducing the following function

$$
\begin{aligned}
J(\theta, \phi, \psi) & =\|\mathbf{Q}(\theta, \phi, \psi) * \mathbb{F}-\mathbb{F}\| \\
& =\|\mathbf{Q}(\theta, \phi, \psi) \cdot \tilde{\mathbf{F}} \cdot \tilde{\mathbf{Q}}(\theta, \phi, \psi)-\tilde{\mathbf{F}}\|
\end{aligned}
$$

where $\mathbf{Q}(\theta, \phi, \psi)$ is a rotation operator parametrized with the longitude, colatitude and rotation angles; $\tilde{\mathbf{Q}}(\theta, \phi, \psi)$ is a $18 \times 18$ matrix whose components are defined by (23), the vanishing loci of $\mathrm{J}(\theta, \phi, \psi)$ allow us to obtain the invariant directions $\mathbf{n}$ that $\mathbb{F}$ possesses.

We illustrate, in Figure 2 the vanishing loci of the function $J(\theta, \phi, 2 \pi / 3)$ plotted on the $\theta-\phi$ plane for a flexoelectric tensor $\mathbb{F}$ belonging to the rotational symmetry class $\left\{Z_{3}\right\}$ whose matrix representation is provided in Section IV. It can be seen from Figure 2 that there exists only one invariant axis spanned by $\mathbf{n}=\mathbf{e}_{3}$ or $\mathbf{n}=-\mathbf{e}_{3}$. This is in agreement with the fact that the rotational symmetry class $\left\{Z_{3}\right\}$ contains $\mathbf{Q}\left(\mathbf{e}_{3}, 2 \pi / 3\right)$.

By combining the knowledge of the matrix representations of all rotational symmetry classes with pole figures, we can finally identify the rotational symmetry class to which a given flexoelectric tensor belongs. The corresponding identification procedure is summarized in Figure 3 .

\section{CONCLUDING REMARKS}

Flexoelectricity is an electromechanical phenomenon which has a great number of potential applications including energy harvesting, sensors, actuators and biotechnology. A full understanding of the fourth-order flexoelectric tensor is essential not only to the fundamental theory of flexoelectricity but also to all possible applications of flexoelectricity. In the present work, which may be viewed as a continuation of our previous one ${ }^{39}$, compact explicit matrix representations of the flexoelectric tensor have been provided for all the 12 possible rotational symmetry classes, so as to facilitate its use in various situations; the reflection symmetry classes of the flexoelectric tensor have been also determined and shown to be identical to those of the fourth-order elastic tensor; a simple and efficient graphic method for identifying the rotational symmetry and reflection symmetry of a given flexoelectric tensor has been elaborated and illustrated. These results contribute to developing the continuum theory of flexoelectricity and rendering the use of this theory easier in various anisotropic cases. 

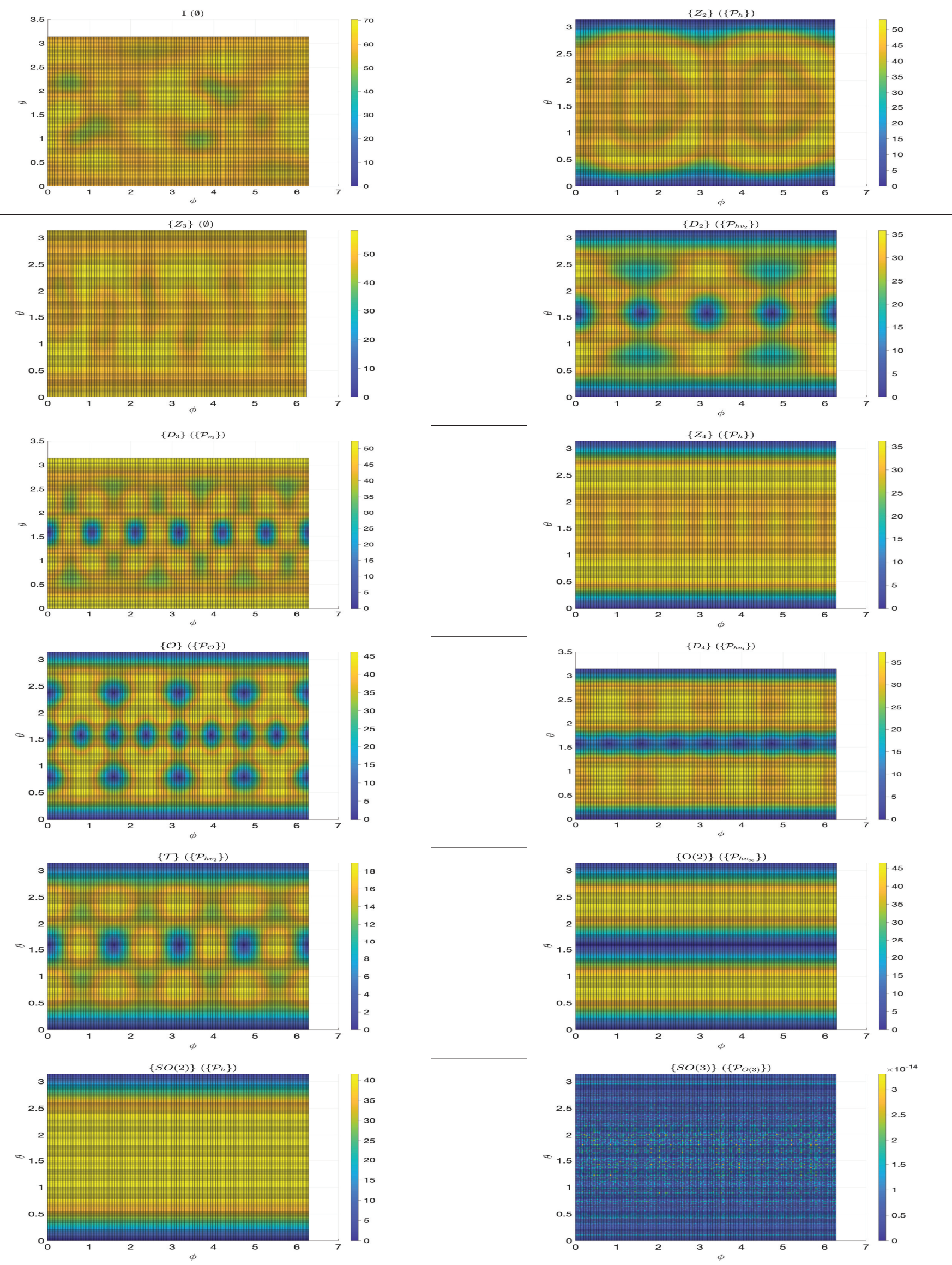

FIG. 1. Loci of the zeros of the function $L(\theta, \phi)$ plotted on the $\theta-\phi$ plane for all rotation symmetry classes and all reflection symmetry classes. 


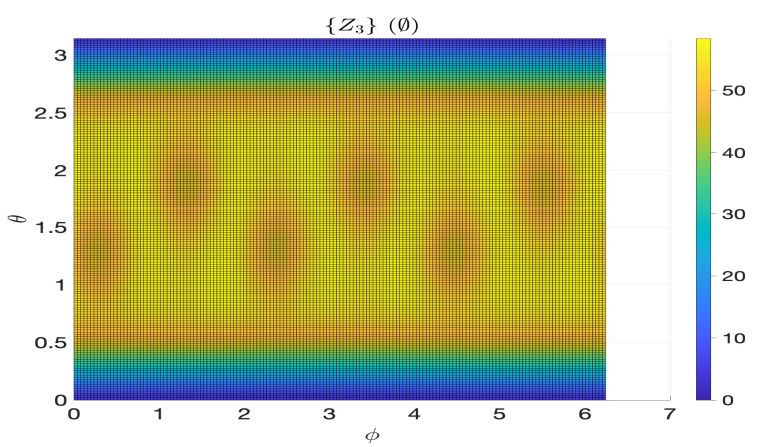

FIG. 2. Loci of the function $J(\theta, \phi, 2 \pi / 3)$ plotted on the $\theta-\phi$ plane for a flexoelectric tensor $\mathbb{F}$ belonging to the rotational symmetry class $\left\{Z_{3}\right\}$
As a simple example of application of our results, we consider Pervoskites with general chemical formula $\mathrm{ABO}_{3}$, which are known as ferroelectric materials exhibiting higher permittivity ferroelectrics like flexoelectricity, piezoelectricity and pyroelectricity than the ones of usual dielectric materials, and which are now widely used for the production of electronic components and microtransducers. Since the oxygen octahedral structure of Pervoskites, the symmetry behavior of the corresponding flexoelectricity tensor is cubic and characterized by the rotation symmetry class $\{\mathcal{O}\}$ or by the reflection symmetry class $\left\{\mathcal{P}_{\mathcal{O}}\right\}$. The matrix of the flexoelectricity tensor relative to an appropriate basis, denoted by $\tilde{\mathbf{F}}_{\mathrm{ABO}_{3}}$, is given by

$\tilde{\mathbf{F}}_{\mathrm{ABO}_{3}}=\left[\begin{array}{cccccccccccccccccc}F_{1111} & F_{2112} & F_{2121} & F_{2112} & F_{2121} & 0 & 0 & 0 & 0 & 0 & 0 & 0 & 0 & 0 & 0 & 0 & 0 & 0 \\ 0 & 0 & 0 & 0 & 0 & F_{1111} & F_{2112} & F_{2121} & F_{2112} & F_{2121} & 0 & 0 & 0 & 0 & 0 & 0 & 0 & 0 \\ 0 & 0 & 0 & 0 & 0 & 0 & 0 & 0 & 0 & 0 & F_{1111} & F_{2112} & F_{2121} & F_{2112} & F_{2121} & 0 & 0 & 0\end{array}\right]$.

The matrix $\tilde{\mathbf{F}}_{\mathrm{ABO}_{3}}$ has 3 independent components, i.e., the longitudinal flexoelectric coefficient $F_{1111}$, transverse flexoelectric coefficient $F_{2112}$ and shear flexoelectric coefficient $F_{2121}$. The values of $F_{1111}, F_{2112}$ and $F_{2121}$ determined experimentally and computationally can be found in Wang et al. ${ }^{34}$ and Shu et al. ${ }^{37}$ for some Pervoskites.

In parallel with the formulation used in the present work in which the electric polarization vector $\mathbf{p}$ is linearly related to the strain gradient $\mathbb{E}$ through the fourth-order flexoelectric tensor $\mathbb{F}$, there is another formulation of the electric polarization vector $\mathbf{p}$ linearly connected to the second-order derivative of the displacement vector, $\mathbb{U}=$ $\nabla \nabla \mathbf{u}$, as follows (see e.g. Hong et al ${ }^{[27}$ ):

$$
p_{i}=F_{i j k l}^{I} U_{j k l} \quad \text { or } \quad p_{i}=F_{i j k l}^{I} u_{j, k l} .
$$

Here $F_{i j k l}^{I}$, the tensor component of the type-I flexoelectric tensor $\mathbb{F}^{I}$, possesses the index permutation symmetry $F_{i j k l}^{I}=F_{i j l k}^{I}$. Compared with $\mathbb{F}$, called also type-II flexoelectric tensor, more suitable not only for formulating the thermodynamic theory of ferroelectric materials but also for making comparisons with experimental measurements, the definition of the type-I flexoelectric tensor $\mathbb{F}^{I}$ is complicated for mathematical derivations of the microscopic theory of flexoelectricity. It can be shown that the connections between $\mathbb{F}^{I}$ and $\mathbb{F}$ are given by

$$
F_{i j k l}^{I}=\frac{1}{2}\left(F_{i j k l}+F_{i j l k}\right), \quad F_{i j k l}=F_{i j k l}^{I}+F_{i k j l}^{I}-F_{i l j k}^{I} .
$$

In addition, due to the fact that both flexoelectric tensors $\mathbb{F}^{I}$ and $\mathbb{F}$ exhibit mathematically the same permutation symmetry with respect to two indexes, the flexoelectric tensors $\mathbb{F}^{I}$ and $\mathbb{F}$ will possess the same rotation and reflection symmetry classifications. Moreover, by adopting
TABLE VII. Suffix notation correspondences between $(j, k, l)$ and $\gamma$.

\begin{tabular}{cc}
\hline \hline$(j, k, l)$ & $\gamma$ \\
\hline$(1,1,1)$ & 1 \\
$(1,2,2)$ & 2 \\
$(2,1,2)$ or $(2,, 2,1)$ & 3 \\
$(1,3,3)$ & 4 \\
$(3,1,3)$ or $(3,3,1)$ & 5 \\
$(2,2,2)$ & 6 \\
$(2,1,1)$ & 7 \\
$(1,1,2)$ or $(1,2,1)$ & 8 \\
$(2,3,3)$ & 9 \\
$(3,2,3)$ or $(3,3,2)$ & 10 \\
$(3,3,3)$ & 11 \\
$(3,1,1)$ & 12 \\
$(1,1,3)$ or $(1,3,1)$ & 13 \\
$(3,2,2)$ & 14 \\
$(2,2,3)$ or $(2,3,2)$ & 15 \\
$(3,1,2)$ or $(3,2,1)$ & 16 \\
$(2,1,3)$ or $(2,3,1)$ & 17 \\
$(1,2,3)$ or $(1,3,2)$ & 18 \\
\hline \hline
\end{tabular}

the reduced suffix notations described in Table VII the fourth-order flexoelectric tenor $\mathbb{F}^{I}$ with component $F_{i j k l}^{I}$ can be expressed in a $3 \times 18$ matrix form $\tilde{\mathbf{F}}^{I}$ with components $\tilde{F}_{i \gamma}^{I}$. Consequently, the number of independent material parameters and compact explicit matrix expression for each of the 12 symmetry classes of flexoelectric tensors $\mathbb{F}^{I}$ are exactly identical to the ones of $\mathbb{F}$.

Finally, it is interesting and important to remark that the methods and results presented in the present work are directly applicable to the fourth-order flexomagnetic tensor ${ }^{46}$. 


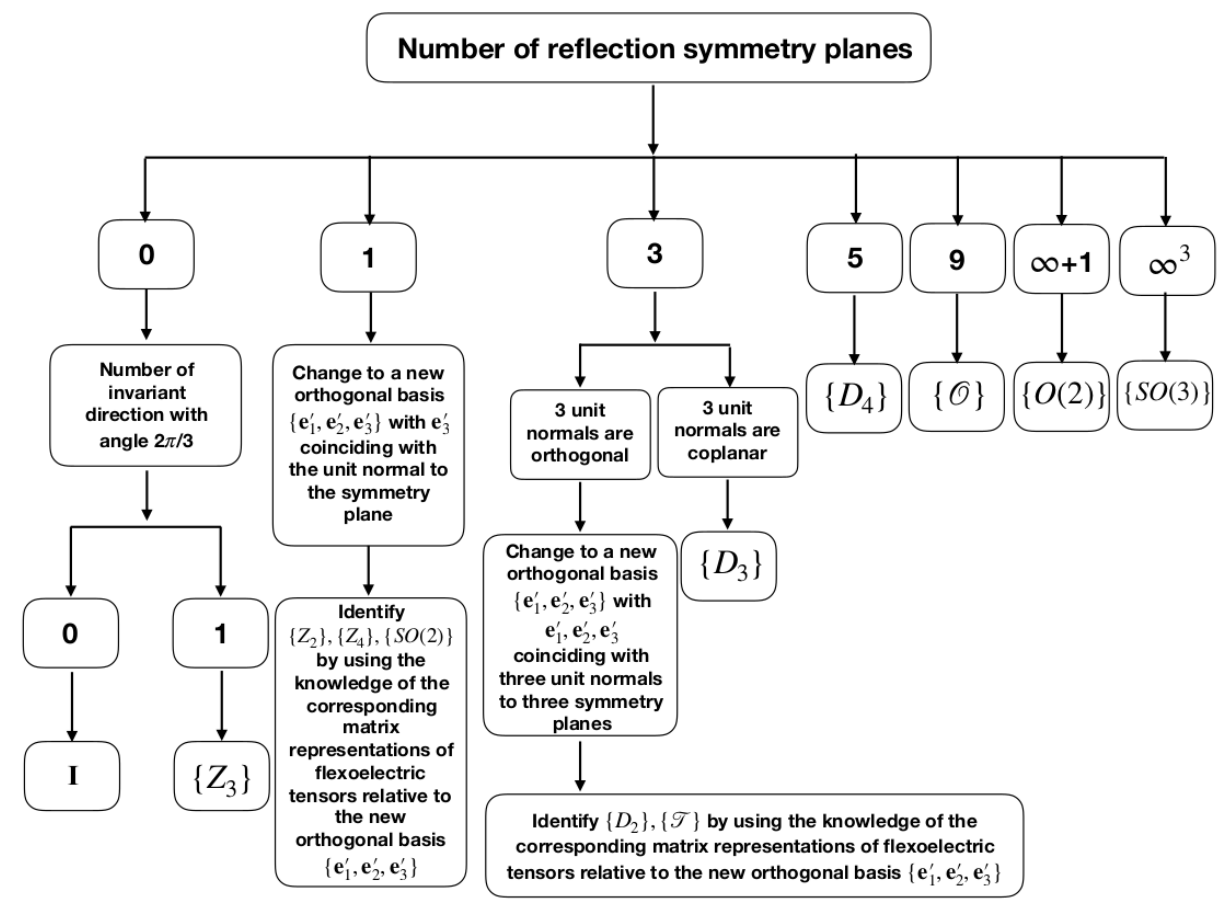

FIG. 3. Procedure for identifying the rotational symmetry class to which a flexoelectric tensor belongs with respect to a given basis

\section{DATA AVAILABILITY}

The data that support the findings of this study are available from the corresponding author upon reasonable request.

\section{REFERENCES}

${ }^{1}$ E. V. Bursian and N. N. Trunov (1974). "Nonlocal piezoelectric effect," Fiz. Tverd. Tela (Leningrad), 16. 1187.

${ }^{2}$ C. Liu, S. Hu and S. Shen (2012). "Effect of flexoelectricity on electrostatic potential in a bent piezoelectric nanowire," Smart Mater. Struct. 21. 115024, Dataset.https://doi.org/10.1088/ 0964-1726/21/11/115024

${ }^{3}$ T. D. Nguyen, S. Mao, Y.-W. Yeh, P. K. Purohit and M. C. McAlpine (2013). "Nanoscale flexoelectricity," Adv. Mater. 25. 946. Dataset. https://doi.org/10.1002/adma. 201203852

${ }^{4} \mathrm{Z}$. Yan and L. Y. Jiang (2013). "Flexoelectric effect on the electroelastic responses of bending piezoelectric nanobeams," J. Appl. Phys. 113. 194103. Dataset. https://doi.org/10.1063/1. 4804949

${ }^{5}$ G. Catalan, L. J. Sinnamon and J. M. Gregg (2004). "The effect of flexoelectricity on the dielectric properties of inhomogeneously strained ferroelectric thin films," J. Phys. Condens. Matter. 16. 2253. Dataset. https://doi.org/10.1088/0953-8984/ $16 / 13 / 006$

${ }^{6}$ R. B. Meyer (1969). "Piezoelectric effects in liquid crystals," Phys. Rev. Lett. 22. 918. Dataset. https://doi.org/10.1103/ PhysRevLett.22.918

${ }^{7}$ M. Marvan and A. Havranek (1988). "Flexoelectric effect in elastomers," Prog. Colloid. Polym. Sci. 78. 33. Dataset. https: //doi.org/10.1007/BFb0114342
${ }^{8}$ W. Ma and L. E. Cross (2001). "Observation of the flexoelectric effect in relaxor $\mathrm{Pb}\left(\mathrm{Mg}_{1 / 3} \mathrm{Nb}_{2 / 3}\right) \mathrm{O}_{3}$ ceramics," Appl. Phys. Lett. 78. 2920. Dataset. https://doi.org/10.1063/1.1356444

${ }^{9} \mathrm{~W}$. Ma and L. E. Cross (2002). "Flexoelectric polarization of barium strontium titanate in the paraelectric state," Appl. Phys. Lett. 81. 3440. Dataset https://doi.org/10.1063/1.1518559

${ }^{10} \mathrm{~W}$. Ma and L. E. Cross (2006). "Flexoelectricity of barium titanate," Appl. Phys. Lett. 88. 232902. Dataset. https://doi. org/10.1063/1.2211309

${ }^{11}$ P. Zubko, G. Catalan, P. R. L. Welche, A. Buckley and J. F. Scott (2007). "Strain-gradient-induced polarization in $\mathrm{SrTiO}_{3}$ single crystals,", Phys. Rev. Lett. 99. 167601. Dataset. https://doi. org/10.1103/PhysRevLett.99.167601

${ }^{12} \mathrm{~S}$. V. Kalinin and V. Meunier (2008). "Electronic flexoelectricity in low-dimensional systems,"Phys. Rev. B 77. 033403. Dataset. https://doi.org/10.1103/PhysRevB.77.033403

${ }^{13}$ I. Naumov, A. M. Bratkovsky and V. Ranjan (2009). "Unusual flexoelectric effect in two-dimensional noncentrosymmetric $\mathrm{sp}^{2}$ bonded crystals," Phys. Rev. Lett. 102. 217601. Dataset. https: //doi.org/10.1103/PhysRevLett.102.217601

${ }^{14}$ S. Zhang, X. Liang, M. Xu, B. Feng and S. Shen (2015). "Shear flexoelectric response along 3121 direction in polyvinylidene fluoride," Appl. Phys. Lett. 107. 142902, Dataset.https://doi.org/ 10.1063/1.4932523

${ }^{15}$ S. Zhang, K. Liu. M. Xu, H. Shen, K. Chen B. Feng and S. Shen (2017). "Investigation of the 2312 flexoelectric coefficient component of polyvinylidene fluoride: Deduction, simulation, and mensuration," Sci. Rep. 7. 3134, Dataset. https: //doi.org/10.1038/s41598-017-03403-7

${ }^{16}$ B. Chu and D.R. Salem (2012). "Flexoelectricity in several thermoplastic and thermosetting polymers," Appl. Phys. 101. 103905, Dataset. https://doi.org/10.1109/TDEI.2017.006273

${ }^{17}$ Y. Zhou, J. Liu and X.P. Hu (2017). "Flexoelectric effect in PVDF-based polymers," IEEE Trans. Dielect. El. 24. 727, Dataset. https://doi.org/10.1109/TDEI.2017.006273

${ }^{18}$ E. Sahin and S. Dost (1998); "A strain-gradients theory of elastic dielectrics with spatial dispersion," Int. J. Eng. Sci. 26. 1231. 
Dataset. https://doi.org/10.1016/0020-7225(88)90043-2.

${ }^{19}$ A. K. Tagantsev (1986). "Piezoelectricity and flexoelectricity in crystalline dielectrics," Phys. Rev. B 34. 5883. Dataset. https: //doi.org/10.1103/PhysRevB.34.5883

${ }^{20} \mathrm{~A}$. K. Tagantsev (1991). "Electric polarization in crystals and its response to thermal and elastic perturbation," Phase Transit. 35. 119. Dataset. https://doi.org/10.1080/01411599108213201

${ }^{21}$ A. S. Yurkov and A. K. Tagantsev (2016). "Strong surface effect on direct bulk flexoelectric response in solids," Appl. Phys. Lett. 108. 022904, Dataset. https://doi.org/10.1063/1.4939975

${ }^{22}$ B. He, B. Javvaji and X.Y. Zhuang (2018). "Size dependent flexoelectric and mechanical properties of barium titanate nanobelt: A molecular dynamics study," Physica B. 545. 527, Dataset. https://doi.org/10.1016/j.physb.2018.01.031

${ }^{23}$ L. Qi, S.J. Zhou and A.Q. Li (2016). "Size-dependent bending of an electro-elastic bilayer nanobeam due to flexoelectricity and strain gradient elastic effect," Compos. Struct. 135. 167, Dataset. https://doi.org/10.1016/j.compstruct.2015.09.020

${ }^{24}$ G. Bai, K. Qin, Q.Y. Xie, X. Yan, C. Gao and Z. Liu (2017). "Size dependent flexocaloric effect of paraelectric $\mathrm{Ba}_{0.67} \mathrm{Sr}_{0.33} \mathrm{TiO}_{3}$ nanostructures," Mater. Lett. 186. 146, Dataset. https://doi. org $/ 10.1016 / \mathrm{j}$.matlet.2016.10.001

${ }^{25} \mathrm{R}$. Maranganti and P. Sharma (2009). "Atomistic determination of flexoelectric properties of crystalline dielectrics," Phys. Rev. B 80. 054109. Dataset. https://doi.org/10.1103/PhysRevB.80. 054109

${ }^{26}$ J.W. Hong and D. Vanderbilt (2011). "First-principles theory of frozen-ion flexoelectricity," Phys. Rev. B 84. 180101, Dataset. https://doi.org/10.1103/PhysRevB.84.180101

${ }^{27}$ J.W. Hong and D. Vanderbilt (2013). "First-principles theory and calculation of flexoelectricity," Phys. Rev. B 88. 174107, Dataset. https://doi.org/10.1103/PhysRevB.88.174107

${ }^{28}$ F. Deng, Q. Deng and S.P. Shen (2018). "A three-dimensional mixed finite element for flexoelectricity," J. Appl. Mech. 85. 031009, Dataset.https://doi.org/10.1115/1.4038919

${ }^{29}$ J. Yvonnet, X. Chen and P. Sharma (2020). "Apparent flexoelectricity due to heterogeneous piezoelectricity," J. Appl. Mech. 87, 111003. Dataset. https://doi.org/10.1115/1.4047981

${ }^{30}$ Q. Li, C.T. Nelson, S.-L. Hsu, A.R. Damodaran, L.-L. Li, A.K. Yadav, M. McCarter, L.W. Martin, R. Ramesh and S.V. Kalinin (2017) "Quantification of flexoelectricity in $\mathrm{PbTiO}_{3} / \mathrm{SrTiO}_{3}$ superlattice polar vortices using machine learning and phase-field modeling," Nat. Commun. 8. 1468, Dataset. https://doi.org/ 10.1038/s41467-017-01733-8

${ }^{31}$ Y.-J. Wang, J. Li, Y.-L. Zhu and X.-L. Ma (2017). "Phase-field modeling and electronic structural analysis of flexoelectric effect at $180^{\circ}$ domain walls in ferroelectric $\mathrm{PbTiO}_{3}, " \mathrm{~J}$. Appl. Phys. 122. 224101, Dataset. https://doi.org/10.1063/1.5017219

${ }^{32}$ N. D. Sharma, R. Maranganti and P. Sharma (2007). "On the possibility of piezoelectric nanocomposites without using piezoelectric materials," J. Mech. Phys. Solids 55. 2328. Dataset. https://doi.org/10.1016/j.jmps.2007.03.016.
${ }^{33}$ P. Zubko, G. Catalan and A. K. Tagantsev (2013). "Flexoelectric effect in solids," Annu. Rev. Mater. Res. 43. 387. Dataset.https : //doi.org/10.1146/annurev-matsci-071312-121634

${ }^{34}$ B. Wang, Y. Gu, S. Zhang and L.-Q. Chen (2019). "Flexoelectricity in solids: Progress, challenges, and perspectives," Prog. Mater. Sci. 106. 100570. Dataset. https://doi.org/10.1016/j. pmatsci.2019.05.003

${ }^{35}$ J. Narvaez, F. Vasquez-Sancho and G. Catalan (2016). "Enhanced flexoelectric-like response in oxide semiconductors," Nature 538. 219, Dataset. https://doi.org/10.1038/nature19761

${ }^{36}$ A. Abdollahi, F. Vasquez-Sancho and G. Catalan (2018). "Piezoelectric Mimicry of Flexoelectricity," Phys. Rev. Lett. 121. 205502, Dataset. https://doi.org/10.1103/PhysRevLett.121. 205502

${ }^{37}$ L. Shu, R. Liang, Z. Rao, L. Fei, S. Ke and Y. Wang (2019). "Flexoelectric materials and their related applications: A focused review," J. Adv. Ceram. 8. 153, Dataset. https://doi.org/10. 1007/s40145-018-0311-3

${ }^{38}$ L. Shu, S. Ke, L. Fei, W. Huang, Z. Wang, J. Gong, X. Jiang, L. Wang, F. Li, S. Lei, Z. Rao, Y. Zhou, R.-K. Zheng, X. Yao, Y. Wang, M. Stengel and G. Catalan (2020). "Photoflexoelectric effect in halide perovskites," Nat. Mater. 19. 605, Dataset.https : //doi.org/10.1038/s41563-020-0659-y

${ }^{39} \mathrm{H}$. Le Quang and Q.-C. He (2011). "The number and types of all possible rotational symmetries for flexoelectric tensors," Roy. Soc. London, Ser. A 467. 2369. Dataset. https://doi.org/10. 1098/rspa.2010.0521

${ }^{40}$ L. Shu, X. Wei, T. Pang, X. Yao and C. Wang (2011). "Symmetry of flexoelectric coefficients in crystalline medium," J. Appl. Phys. 110. 104106. Dataset. https://doi.org/10.1063/1.3662196

${ }^{41} \mathrm{~A}$. Spencer (1970). "A note on the decomposition of tensors into traceless symmetric tensors," Int. J. Engng. Sci. 8. 475. Dataset. https://doi.org/10.1016/0020-7225(70)90024-8

${ }^{42}$ S. Forte and M. Vianello (1996). "Symmetry classes for elasticity tensors," J. Elasticity 43. 81. Dataset. https://doi.org/10. $1007 / \mathrm{BF} 00042505$

${ }^{43}$ S. Forte and M. Vianello (1997). "Symmetry classes and harmonic decomposition for photoelasticity tensors," Int. J. Engng. Sci. 14. 1317. Dataset. https://doi.org/10.1016/ S0020-7225(97) 00036-0

${ }^{44} \mathrm{P}$. Chadwick, M. Vianello and S. Cowin (2001). "A new proof that the number of linear anisotropic elastic symmetries is eight," J. Mech. Phys. Solids 49. 2471. Dataset. https://doi.org/10. 1016/S0022-5096(01) 00064-3

${ }^{45}$ M. Francois, G. Geymonat and Y. Berthaud (1998). "Determination of the symmetries of an experimentally determined stiffness tensor: Application to acoustic measurements," Int. J. Solids Struct. 35. 4091. Dataset. https://doi.org/10.1016/ S0020-7683(97) 00303-X

${ }^{46}$ P. Lukashev and R. F. Sabirianov (2010). "Flexomagnetic effect in frustrated triangular magnetic structures," Phys. Rev. B 82. 094417. Dataset.https://doi.org/10.1103/PhysRevB.82. 094417. 